\title{
Avaliação de Degradação de Estruturas de Concreto em Duas Garagens de Edifícios Residenciais pelo Método GDE/UnB
}

\section{Evaluation of Concrete Structures' Degradation in Two Garages of Residential Buildings by the GDE / UnB Method}

\author{
Rafael Rocha GALVÃO ${ }^{1}$, Francisco Gabriel Santos SILVA ${ }^{2}$ \\ ${ }^{1}$ Universidade Federal da Bahia, Escola Politécnica, Brasil, rafaelrgalvao.eng@gmail.com \\ 2 Universidade Federal da Bahia, Escola Politécnica - Departamento de Construção e Estruturas, Brasil, \\ fgabriel.ufba@gmail.com
}

\begin{abstract}
Resumo: Até o final dos anos 60, era comum o lançamento de empreendimentos residenciais e comerciais sem a existência de garagens, mas com a popularização dos automóveis, as garagens tornaram-se um item indispensável para qualquer edificação e a sua ausência é causa de desvalorização. Por estarem, de maneira geral, na parte inferior do edifício, as garagens estão sujeitas a grandes carregamentos e esforços. A inexistência de manutenções preventivas e periódicas ameaça, com o passar do tempo, a sensação de segurança dos usuários e a integridade das estruturas. O objetivo desse estudo foi realizar, através do método GDE/UnB, a análise sobre a ocorrência de patologias e o respectivo grau de deterioração em estruturas de concreto em duas garagens de edificações residenciais, em Salvador/BA, e comparar os efeitos da maritimidade. Este método consiste em verificar a ocorrência de patologias e danos mais frequentes, a partir de inspeções visuais, dividindo os elementos estruturais em famílias e considerando o respectivo grau de intensidade de cada uma, chegando a um valor final de deterioração da estrutura observada. 0 estudo de caso foi realizado em dois prédios com mais de 40 anos de construção e com ausência de manutenções: 0 Edifício 1, mais próximo ao mar, e o Edifício 2, numa região mais central e um pouco mais distante do mar. Apesar de o Edifício 2 ter um certo percentual de elementos em nível crítico, foi possível ratificar a hipótese levantada inicialmente: o Edifício 1, por estar, mais próximo ao mar, teria um percentual maior de ocorrência de patologias, com grande presença de manchas, umidade, corrosão de armaduras, entre outros. De acordo com os resultados encontrados, foram observadas 192 patologias no Edifício 1 contra 91 no Edifício 2, ou seja, cerca de $111 \%$ a mais.
\end{abstract}

Palavras-chave: durabilidade; vida útil; estruturas de concreto; deterioração; metodologia GDE/UnB

Abstract: Until the end of the 1960s, it was common to launch residential and commercial buildings without the existence of garages, but with the popularization of automobiles, garages became an indispensable item for any building and its absence is a cause for devaluation. As garages are generally at the bottom of the building, they are subject to heavy loads and efforts. The lack of preventive and periodic maintenance threatens, over time, the users' feeling of security and the integrity of the structures. The objective of this study was to accomplish, through the GDE / UnB method, the analysis of the occurrence of pathologies and the respective degree of deterioration in concrete structures in two garages of residential buildings, in Salvador / BA, and to compare the effects of maritime conditions. This method consists of verifying the occurrence of pathologies and more frequent damages, with visual inspections, dividing the structural elements into families and considering the respective degree of intensity of each one, reaching a final value of deterioration of the observed structure. The case study was realized in two buildings with more than 40 years of construction and with no maintenance: Building 1, closer to the sea, and Building 2, in a more central region and a little further from the sea. Although Building 2 has a certain percentage of elements at a critical level, it was possible to ratify the hypothesis initially raised: Building 1, being closer to the sea, would have a higher percentage of the occurrence of pathologies, with a high presence of stains, moisture, reinforcement corrosion, among others. According to the results found, 192 pathologies were observed in Building 1 against 91 in Building 2, that is, about $111 \%$ more.

Key-words: durability; lifespan; concrete structures; deterioration; GDE / UnB methodology 


\section{Introdução}

O concreto é o material construtivo mais consumido do mundo, segundo pesquisa realizada, em 2012, pela Associação Brasileira de Cimento Portland (ABCP). Para garantir um melhor desempenho e uma maior vida útil, cuidados especiais são necessários, desde a seleção de materiais até a manutenção dos componentes executados. Contudo, ao longo da sua vida, as edificações e demais tipos estruturais estão sujeitos à ação de diversos agentes agressivos que impactam na redução da resistência e durabilidade da construção.

O presente trabalho tem como objetivo realizar o estudo sobre a ocorrência de patologias e o respectivo grau de deterioração em estruturas de concreto, com uso da metodologia de inspeções visuais Gde/UnB, em garagens de edificações residenciais de diferentes localidades de Salvador/BA, analisando a influência marítima no processo de degradação.

\subsection{Vida Útil e Durabilidade}

Como as estruturas estão sujeitas às ações de diversos tipos, seja devido a ações de cargas e sobrecargas, a NBR 6118:2014 apresenta o conceito de vida útil, que é o período de tempo durante o qual as estruturas mantêm as suas características, sem intervenções significativas, ressaltando que os requisitos de uso e manutenção prescritos pelo projetista e construtor, juntamente com os reparos necessários decorrentes de eventuais danos acidentais, devem ser mantidos.

Para a NBR 6118:2014, no item 5.1.2.3, a durabilidade é a capacidade de a estrutura resistir às influências ambientais previstas e definidas, no ato de elaboração dos projetos, entre o autor do projeto estrutural e o contratante. As estruturas, sob tais influências, devem conservar sua segurança, estabilidade e possuir comportamento adequado em serviço durante o período corresponde à sua vida útil de projeto.

Segundo o item 6.4 da supracitada NBR, um dos principais responsáveis pela perda de qualidade e durabilidade das estruturas é a agressividade do meio ambiente, que está relacionada às ações físicas e química que atuam sobre as estruturas de concreto, sem dependência das ações mecânicas, das variações volumétricas de origem térmica, da retração hidráulica e outros fatores previstos no dimensionamento. A norma apresenta a tabela 1, na qual, a agressividade ambiental pode ser avaliada, simplificadamente, segundo as condições de exposição da estrutura ou de suas partes, no projeto de estruturas correntes.

Tabela 1 - Classes de agressividade ambiental (CAA)

\begin{tabular}{|c|c|c|c|}
\hline $\begin{array}{l}\text { Classe de Agressividade } \\
\text { Ambiental }\end{array}$ & Agressividade & $\begin{array}{l}\text { Classificação geral quanto ao tipo } \\
\text { de ambiente para efeito de projeto }\end{array}$ & $\begin{array}{c}\text { Risco de deterioração } \\
\text { da estrutura }\end{array}$ \\
\hline \multirow{2}{*}{1} & \multirow{2}{*}{ Fraca } & Rural & \multirow{2}{*}{ Insignificante } \\
\hline & & Submersa & \\
\hline II & Moderada & Urbana & Pequeno \\
\hline \multirow{2}{*}{ III } & \multirow{2}{*}{ Forte } & Marinha & \multirow{2}{*}{ Grande } \\
\hline & & Industrial & \\
\hline \multirow{2}{*}{ IV } & \multirow{2}{*}{ Muito Forte } & Industrial & \multirow{2}{*}{ Elevado } \\
\hline & & Respingos de maré & \\
\hline
\end{tabular}

Fonte: NBR 6118 (2014)

\subsection{Patologias e Danos mais frequentes em Estruturas de Concreto}

A Patologia das Estruturas pode ser entendido como o campo de pesquisa destinado a estudar as origens, manifestações, consequências e os mecanismos de falhas provocados por degradações nas estruturas. $O$ termo "patologia", extraído da Medicina, é usado na construção civil, com o objetivo de definir as "doenças" que acometem as estruturas, fazendo uma analogia ao comparar a edificação ao corpo humano (SOUZA E RIPPER, 1998). 
As patologias podem ser classificadas em simples ou complexas, de acordo com análise do diagnóstico e profilaxia. Os danos variam desde uma menor escala, causando um simples desconforto ao usuário final, até grandes proporções, levando a estrutura ao colapso (HELENE, 1992). A figura 1 mostra as manifestações mais comuns em estruturas de concreto.

Figura 1 - Distribuição Relativa da Incidência de Manifestações Patológicas em Estruturas de Concreto Aparente

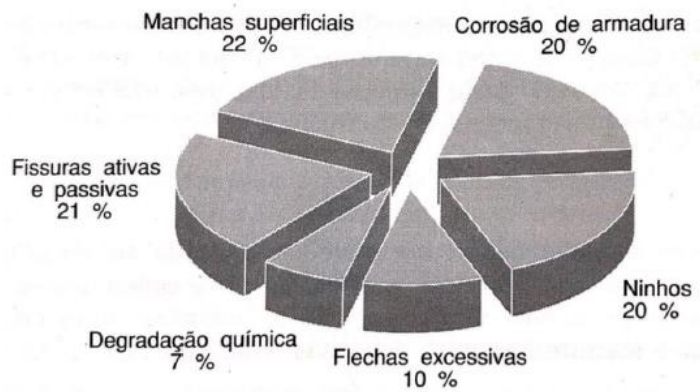

Fonte: Helene (1992)

Souza e Ripper (1998) classificam as causas das manifestações patológicas em intrínsecas e extrínsecas. As primeiras são decorrentes de processos de deterioração das estruturas de concreto as que são inerentes às próprias estruturas, como materiais de baixa qualidade ou erros na execução. Já as segundas "são as que independem do corpo estrutural em si, assim como da composição interna do concreto, ou de falhas inerentes ao processo de execução, podendo, de outra forma, ser vistas como os fatores que atacam a estrutura 'de fora para dentro', durante as fases de concepção ou ao longo da vida útil desta".

\subsection{Degradação de Estruturas em Salvador/BA}

Uma pesquisa realizada por Vilasboas (2004), analisando 48 estruturas de concreto armado na cidade de Salvador/BA, em conjunto com a opinião de projetistas, empresas de recuperação de estruturas e fornecedores de concreto, constatou que a despassivação da armadura pela ação da carbonatação ou íon cloro é responsável por grande parte da causa de patologias em edificações na capital baiana, estando presente em $52 \%$ das estruturas observadas. Outro fator determinante para o aparecimento de patologias, entre as citadas anteriormente também, é o cobrimento deficiente (falha de projeto ou execução), verificado em $56,25 \%$ dos itens (VILASBOAS, 2004).

Ainda segundo a pesquisa, $88,2 \%$ dos profissionais que colaboram com a pesquisa reconhecem que a classe de agressividade ambiental utilizadas nos projetos (moderada ou forte) é inadequada quando a edificação se encontra a $400 \mathrm{~m}$ ou menos da orla marítima. Dessa forma, Vilasboas (2004) propõe uma nova classificação de agressividade ambiental, utilizando o mapa de Salvador, no qual subdivide as regiões de acordo com o seu grau de intensidade, a partir das estruturas estudadas, conforme pode ser visto na figura 2.

Figura 2 - Classe de Agressividade Ambiental de acordo com a região, em Salvador/BA

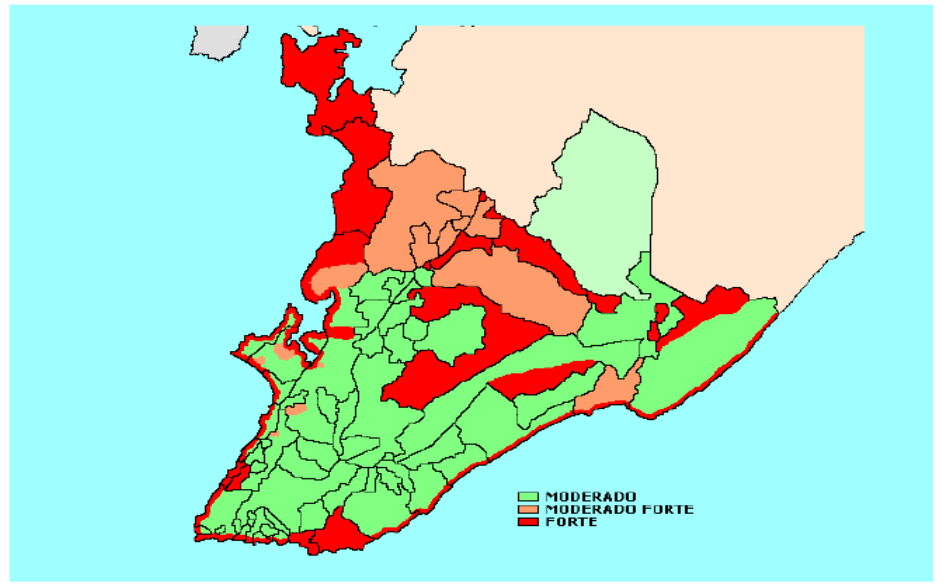

Fonte: Vilasboas (2004) 


\section{Metodologia}

A metodologia utilizada para aplicação no estudo de caso é a GDE/UnB, adaptada de Fonseca (2007), para quantificar o grau de deterioração de estruturas de concreto armado presentes em garagens de edificações, na cidade de Salvador/BA, através de um roteiro elaborado a partir de inspeções visuais. O método original foi proposto por Castro (1994), com o objetivo de avaliar, quantificar e dar criticidade às estruturas de concreto armado convencionais, baseando-se em parâmetros que consideram as manifestações mais frequentes de danos, sua evolução e a influência do meio ambiente em que a estrutura está inserida e, assim, realçando a definição de prioridades para realização de manutenções. O fluxograma da figura 2 ilustra as etapas para aplicação da metodologia GDE/UnB, adaptada de Fonseca (2007), em estruturas convencionais de concreto armado.

Figura 3 - Fluxograma para aval. de degradação de estruturas de concreto armado através da metodologia GDE/UnB

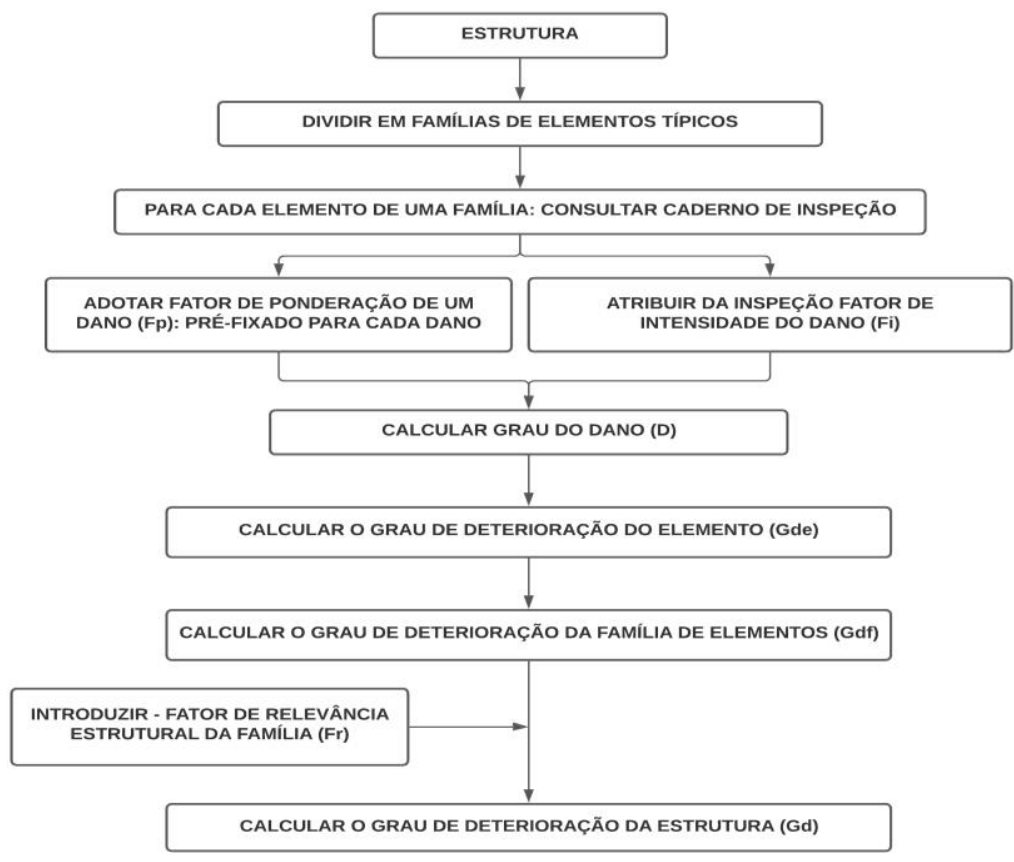

Fonte: Fonseca (2007)

O roteiro proposto por Fonseca (2007) é caracterizado pelo preenchimento de uma ficha de inspeção, coletando-se, inicialmente, dados do objeto de estudo, como localização, natureza de uso, idade, sistema construtivo e classe de agressividade ambiental. A sequência de etapas consiste em: identificação dos elementos estruturais, registro fotográfico, cálculo do Grau do Dano (D), o grau de deterioração do elemento (Gde), o grau de deterioração de uma família de elementos (Gdf) e, por fim, o grau do grau de deterioração da estrutura (Gd), com auxílio do fator de ponderação dos danos ( $F p)$, do fator de intensidade dos mesmos (Fi) e do fator de relevância estrutural (Fr). Por fim, tem-se a classificação dos níveis de deterioração, conforme tabela 2 .

Tabela 2 - Classificação dos níveis de deterioração

\begin{tabular}{c|c|l}
\hline Nível de deterioração & Gde/Gd & \multicolumn{1}{|c}{ Ações a serem adotadas } \\
\hline Baixo & $0-15$ & Estado aceitável. Manutenção preventiva. \\
\hline Médio & $15-50$ & intervenção em longo prazo (máx. 2 anos). \\
\hline Alto & $50-80$ & Planejar intervenção em médio prazo (máx. 1 ano). \\
\hline Sofrível & $80-100$ & Planejar intervenção em curto prazo (máx. 6 meses). \\
\hline Crítico & $>100$ & Inspeção especial emergencial. Planejar intervenção imediata. \\
\hline
\end{tabular}

Fonte: Fonseca (2007) 


\section{Estudo de Caso}

Foram escolhidas duas garagens de prédios residenciais de idades e características semelhantes, mas com a principal diferença relacionada a sua localização. A primeira, do Edifício Solar Itatiaia, situada no Rio Vermelho, e a segunda, do edifício Nossa Senhora do Loreto, nos Barris. Em ambas as localidades, foram realizadas inspeções visuais, considerando os fatores ambientais, com registro fotográfico e mapeamento das estruturas. Também, fez-se o levantamento de croqui, juntamente com a observação de patologias e a avaliação de grau de danos.

\subsection{Edifício Solar Itatiaia}

Figura 4 - Edifício Solar Itatiaia

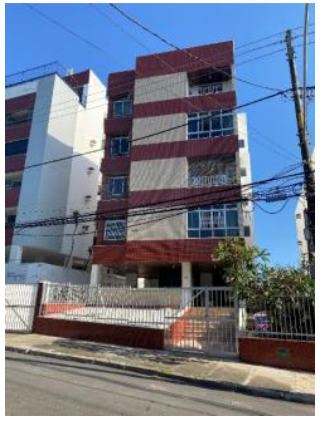

- Localizado no Rio Vermelho, a cerca de $500 \mathrm{~m}$ da orla;

- Idade estimada de 42 anos;

- 6 pavimentos;

- Classe de Agressividade III Forte - Marinha;

- Ausência de Projetos Estruturais.

Fonte: O autor (2020)

\subsection{Edifício São Felipe}

Figura 5 - Edifício São Felipe

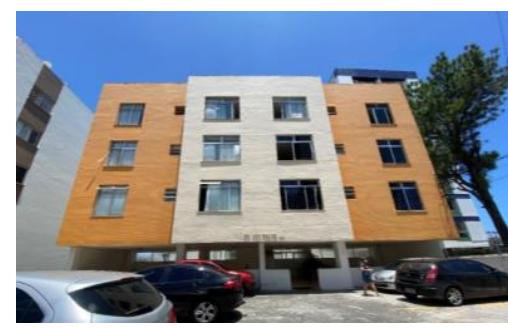

Fonte: $O$ autor (2020)
- Localizado na Federação, a 1,65 km da orla;

- 49 anos de construção;

- 2 torres, uma com 5 andares (estudada) e outra com 6 andares;

- Classe de Agressividade III Forte - Marinha;

- Ausência de Projetos Estruturais.

\subsection{Cálculos do grau do dano e do GDE}

Para cada um dos elementos estruturais das duas garagens, foi elaborada uma tabela, conforme exemplo abaixo, a qual continha os danos existentes nesse elemento, o fator de ponderação desse dano e também o fator de intensidade do dano. Com essas informações, foi possível calcular o grau do dano e o Gde, o grau de deterioração do elemento, e assim avaliar o nível de deterioração existente.

Tabela 3 - Exemplo de tabela utilizada na avaliação das Estruturas

\begin{tabular}{|c|c|c|c|c|}
\hline Nome da Peça & \multicolumn{3}{|c|}{ V18 } & Fotos/Croquis/Observações \\
\hline Local & \multicolumn{3}{|c|}{ Garagem } & \\
\hline Danos & $\mathrm{Fp}$ & $\mathbf{F i}$ & D & \\
\hline Cobrimento deficiente & 3 & 3 & 24 & \\
\hline Corrosão das Armaduras & 5 & 4 & 100 & \\
\hline Desplacamento & 3 & 3 & 24 & \\
\hline \multicolumn{3}{|c|}{ Grau de Deterioração do Elemento (Gde) } & 132,43 & \\
\hline \multicolumn{3}{|c|}{ Nível de Deterioração da Peça } & CRÍTICO & \\
\hline
\end{tabular}

Fonte: O autor (2020) 
Dessa maneira, obteve-se o seguinte resultado para o Ed. Solar Itatiaia, conforme tabela 4:

Tabela 4 - Grau de Deterioração da Estrutura

\begin{tabular}{c|c|c|c|c}
\hline Familia & $G d f$ & $F r$ & $G d f x F r$ & Gd \\
\cline { 1 - 3 } Pilares & 80,97 & 5,00 & 404,85 & 404,90 \\
\cline { 1 - 3 } Vigas & 80,98 & 5,00 & \multirow{2}{*}{$\mathbf{7 9 , 2 6}$} \\
\cline { 1 - 3 } Lajes & 86,11 & 4,00 & 344,44 & 193,14 \\
\hline
\end{tabular}

Fonte: $O$ autor (2020)

Com o resultado de $\mathrm{Gd}$ igual a 79,26, a estrutura analisada recebe a classificação de um grau de deterioração alto, conforme o roteiro de Fonseca (2007) e que é retratado na tabela 5. Já para o Ed. São Felipe, tem-se:

Tabela 5 - Grau de Deterioração da Estrutura

\begin{tabular}{c|c|c|c|c}
\hline Familia & $G d f$ & $F r$ & $G d f \times F r$ & Gd \\
\hline Pilares & 29,39 & 5,00 & 146,95 & 881,25 \\
\cline { 1 - 3 } Vigas Principais & 176,25 & 5,00 & 0 & \multirow{2}{*}{97,61} \\
\cline { 1 - 3 } V. Secundárias & 0 & 4,00 & 338,4 & \\
\hline Lajes & 84,60 & 4,00 & 0 \\
\hline
\end{tabular}

Fonte: O autor (2020)

Com o resultado de Gd igual a 97,61, a estrutura analisada recebe a classificação de um grau de deterioração sofrível, conforme o roteiro de Fonseca (2007).

\section{Resultados e Discussões}

\subsection{Ratificação do Estudo de Vilasboas (2004)}

O estudo de Vilasboas (2004) apresenta um retrato mais fiel da ação do ambiente sobre a durabilidade e vida útil das estruturas. De acordo com classificação dele, o edifício Solar Itatiaia, se encontra em uma classificação "forte", está na categoria "moderado", em uma escala que varia entre moderado, moderado forte e forte.

Apesar de o edifício São Felipe, segundo o método GDE/UnB, ter um grau de deterioração da estrutura "Sofrível", e o Solar Itatiaia, "Alto", foi possível observar que a ocorrência de danos e patologias foi consideravelmente superior no segundo. Foram encontrados, ao todo, 182 patologias e danos em 63 elementos vistoriados no Ed. Solar Itatiaia, o que dá quase 3 patologias por elemento. Boa parte desses danos estão relacionados com a proximidade com o mar, os quais pode-se citar: manchas, umidade, eflorescência, entre outros. Já no Ed. São Felipe, foram observados 91 patologias e danos em 56 elementos estruturais, ou seja, cerca de 1,6 patologia por elemento, valor $44 \%$ inferior ao Solar Itatiaia.

\subsection{Nível de Deterioração das Estruturas}

No Ed. Solar Itatiaia, de modo geral, os graus "baixo" e "médio" representaram 76\% das estruturas analisadas, justamente, como explicado anteriormente, por uma maior incidência de fatores de intensidade 2 e 3. Não foram observados elementos em estado sofrível ou crítico. 
Gráfico 1 - Nível de Deterioração Total dos Elementos

\begin{tabular}{|c|c|c|c|c|c|}
\hline \multirow{3}{*}{$\begin{array}{r}100 \% \\
50 \% \\
0 \%\end{array}$} & $59 \%$ & & & & \\
\hline & 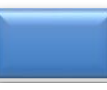 & $17 \%$ & $24 \%$ & $0 \%$ & $0 \%$ \\
\hline & BAIXO & MÉDIO & $\begin{array}{r}\text { ALTO } \\
\text { 甲TOTAL }\end{array}$ & SOFRÍVEL & CRÍTICO \\
\hline
\end{tabular}

Fonte: O autor (2020)

No Ed. São Felipe, considerando a estrutura como um todo, no gráfico 2, o resultado não poderia ser diferente, com "baixo" ocupando $73 \%$ dos elementos. Entretanto, nota-se uma presença de "crítico", puxada por vigas com nível bastante elevado de deterioração, com desplacamento, cobrimento deficiente e ativa corrosão de armaduras.

Gráfico 2 - Nível de Deterioração Total dos Elementos

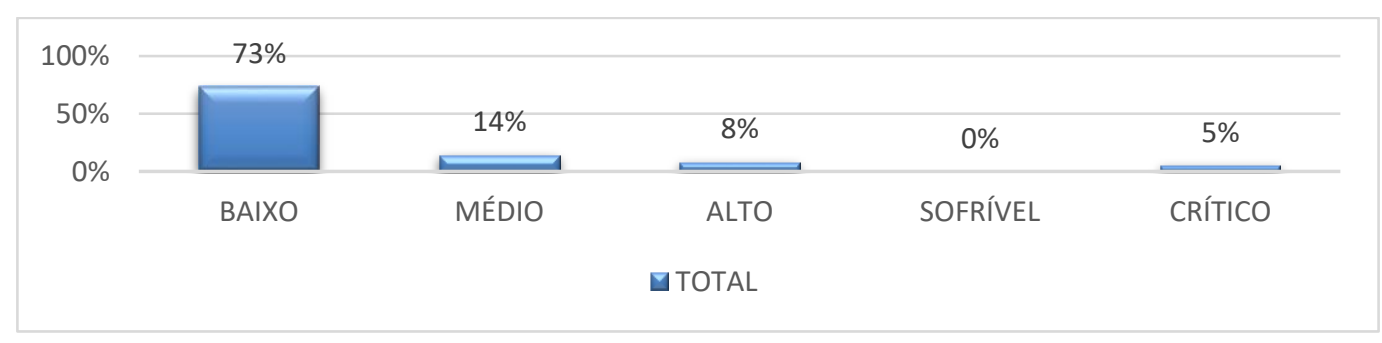

Fonte: O autor (2020)

\subsection{Frequência de Ocorrência das Patologias}

Analisando os gráficos 3 e 4, é perceptível que há uma maior incidência de patologias relacionadas com a proximidade do mar no Ed. Solar Itatiaia. Existe uma grande presença de manchas neste, causadas por fungos originados da umidade. Há também uma presença significativa de ocorrência de "umidade" e "eflorescência", com maior porcentagem no prédio mais próximo à orla marítima, as quais podem ter as prováveis causas relacionadas a penetrações de água em fissuras, que representam um percentual elevado em ambos os edifícios, ou ainda em juntas de concretagem mal executadas, nichos de concretagem ou devido à alta porosidade do concreto. Existe também a possibilidade de estar conectada com uma má impermeabilização da estrutura ou deficiência no escoamento das águas pluviais.

Em ambas as edificações, os danos "cobrimento deficiente" e "corrosão de armaduras" tiveram também uma incidência considerável. Já o segundo dano está bastante relacionado com o primeiro e também com a ocorrência de desplacamento, visto que, com isso, as armaduras acabam ficando expostas ao ambiente e, então, o processo de corrosão passa a ser favorecido e acelerado. Foram constatadas tanto acontecimentos de fissuras de corrosão, de grau de intensidade 3 , como manifestações leves, de intensidade 2 , e corrosão agressiva, de intensidade 4 .

Gráfico 3 - Frequência de Ocorrência de Danos no Ed. Solar Itatiaia

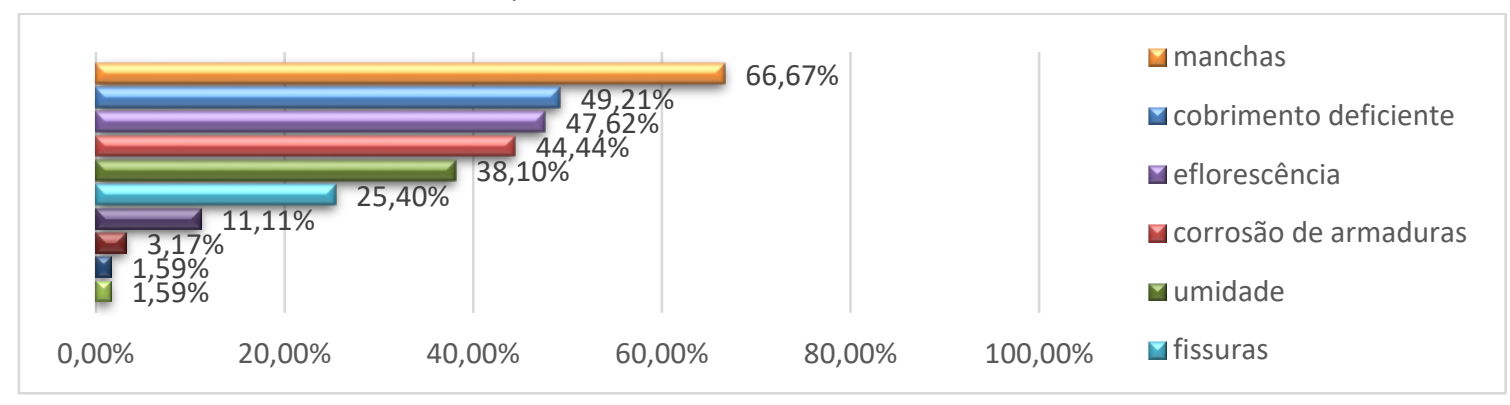

Fonte: O autor (2020) 
Gráfico 4 - Frequência de Ocorrência de Danos no Ed. São Felipe

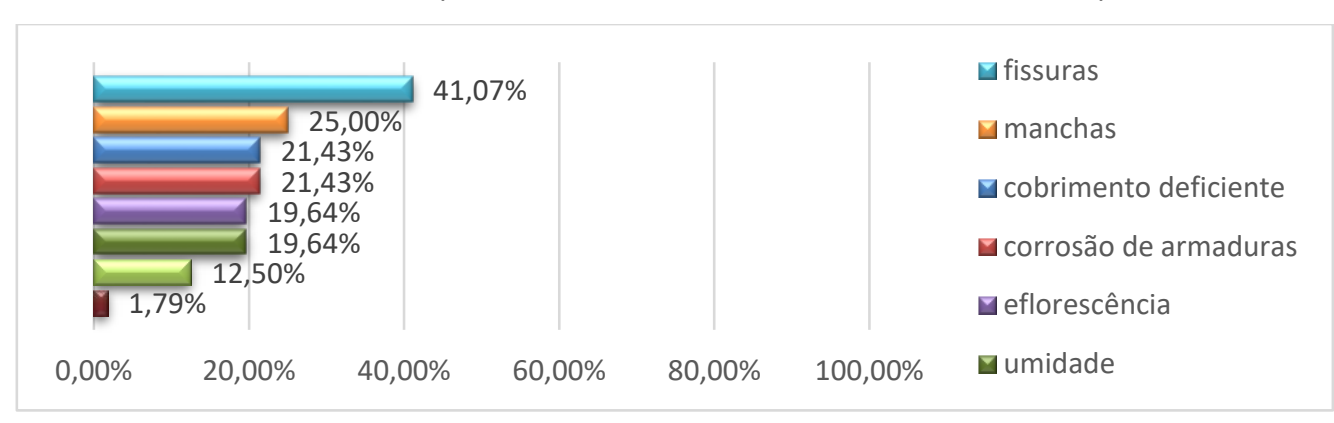

Fonte: O autor (2020)

\section{Conclusões}

Pode-se dizer que o presente trabalho cumpriu com seus objetivos definidos inicialmente, que foi a aplicação da metodologia GDE/UnB, a fim de encontrar o grau de deterioração das estruturas das garagens e analisar quanto aos efeitos da proximidade com o mar. Essa análise permitiu identificar o grau de urgência de manutenção que, de modo geral, para o São Felipe, tem que ser dentro de 6 meses, mas devido à ocorrência de elementos críticos, sugere-se o início imediato e, para o Solar Itatiaia, uma manutenção dentro de 1 ano. Embora o edifício São Felipe tenha o resultado do grau de deterioração da estrutura como "Sofrível", e o Solar Itatiaia, "Alto", foi possível observar que a ocorrência de danos e patologias foi consideravelmente superior no segundo, sendo que uma considerável parte desses danos está relacionada com a proximidade do mar, que pode-se citar: manchas, umidade, eflorescência, entre outros.

No ed. Solar Itatiaia, pôde-se verificar que a maior parte dos pilares e vigas se encontram na faixa entre "baixo" e "médio". As lajes e escada/rampa tiveram grande participação nas categorias "médio" e "alto", fato que se explica devido à ocorrência de várias patologias simultâneas. Já no ed. São Felipe, notável parcela das ocorrências verificadas estão na categoria "baixo", de quase todos os elementos, fato justificável por vários elementos sem ocorrência de patologias visíveis e também pela ocorrência não simultânea dos danos. Entretanto, nota-se uma presença de "crítico", puxada por vigas com nível bastante elevado de deterioração, com desplacamento, cobrimento deficiente e ativa corrosão de armaduras.

Foi notado também a importância das normas e que as exigências atuais sejam cumpridas, na medida que, em muitos casos, foram observadas ocorrências que se originaram, em uma certa parte, por uma falta de rigor das normas antigas. Todas as análises aqui realizadas foram repassadas aos gestores/síndicos dos condomínios estudados e ressaltadas quanto à importância das inspeções periódicas e das manutenções preventivas.

\section{Referências Bibliográficas}

ASSOCIAÇÃO BRASILEIRA DE NORMAS TÉCNICAS (ABNT). NBR 6118 (2014) - Projeto de estruturas de concreto - Procedimento. Rio de Janeiro, 2014.

CASTRO, E. K. Desenvolvimento de metodologia para manutenção de estruturas de concreto armado, Dissertação de Mestrado, Departamento de Engenharia Civil, Universidade de Brasília, Brasília, DF, 1994.

FONSECA, R. P. A estrutura do Instituto Central de Ciências: Aspectos históricos, científicos e tecnológicos de projeto, execução, intervenções e propostas de manutenção. Dissertação de Mestrado, Departamento de Engenharia Civil e Ambiental, Universidade de Brasília, Brasília, DF, 2007.

HELENE, P. R. L. Manual para Reparo, Reforço e Proteção de Estruturas de Concreto. São Paulo: PINI, 1992. SOUZA, V.; RIPPER, T. Patologia, Recuperação e Reforço de Estruturas de Concreto. São Paulo: PINI, 1998.

VILASBOAS, J. M. L. Durabilidade das Edificações de concreto armado em Salvador: uma contribuição para a implantação da NBR 6118:2003 - Dissertação (Mestrado). Departamento de Engenharia Ambiental, Universidade Federal da Bahia, 2004. 